\title{
Role of LncRNAs in regulating cancer amino acid metabolism
}

\author{
Yuhong Guo $0^{1,2,3,4 \dagger}$, Bin Lv ${ }^{1,2,3,4 \dagger}$, Renfeng Liu ${ }^{1,2,3,4}$, Zhengzai Dai ${ }^{1,2,3,4}$, Feifei Zhang ${ }^{1}$, Yiping Liang ${ }^{1}$, Bo Yu $u^{1,2,3,4}$, \\ Duo Zeng ${ }^{1,2,3,4}$, Xiao-Bin LV ${ }^{1 *}$ and Zhiping Zhang ${ }^{1,2,3^{*}}$ (1)
}

\begin{abstract}
The metabolic change of tumor cells is an extremely complicated process that involves the intersection and integration of various signal pathways. Compared with normal tissues, cancer cells show distinguished metabolic characteristics called metabolic reprogramming, which has been considered as a sign of cancer occurrence. With the deepening of tumor research in recent years, people gradually found that amino acid metabolism played crucial roles in cancer progression. Long non-coding RNAs (InCRNAs), which are implicated in many important biological processes, were firstly discovered dysregulating in cancer tissues and participating in extensive regulation of tumorigenesis. This review focuses on the reprogramming of amino acid metabolism in cancers and how IncRNAs participate in the regulatory network by interacting with other macromolecular substances. Understanding the functions of IncRNA in amino acid reprogramming in tumors might provide a new vision on the mechanisms of tumorigenesis and the development of new approaches for cancer therapy.
\end{abstract}

Keywords: IncRNA, Metabolism, Amino acid, Glutamine, Cancer

\section{Background}

The proliferation of normal cells requires the continuous accumulation of substances, so as to produce offspring cells. The accumulated substances in cells include proteins, lipids and nucleic acids, which are essential for cell proliferation [1]. In the past three decades, the studies on oncogenes revealed that the characteristic phenotype of cancer cells is often caused by somatic mutations, and it is the integration of these mutations that lead to the changes of various signal pathways and the state of cell metabolism. The PI3K/AKT/mTOR and the AMPactivated protein kinase pathways are more common

\footnotetext{
*Correspondence: nclvxiaobin@sina.cn; ndsfy001425@ncu.edu.cn

${ }^{\dagger}$ Yuhong Guo and Bin Lv contributedequally to this work

1 Jiangxi Key Laboratory of Cancer Metastasis and Precision Treatment, The Third Affiliated Hospital of Nanchang University, Northern 128 Xiangshan Road, Nanchang 330008, Jiangxi, People's Republic of China ${ }^{2}$ Department of Orthopedics, The Third Affiliated Hospital of Nanchang University, Northern 128 Xiangshan Road, Nanchang 330008, Jiangxi, People's Republic of China

Full list of author information is available at the end of the article
}

dysregulated pathways in cancer [2, 3]. Researches on tumorigenesis have made it clear that these pivotal oncogenic signals lead to the unique metabolic characteristics of tumor cells and support their proliferation. Therefore, the change of cell metabolism is considered as a crucial hallmark in the development of cancer [4].

The occurrence of cancer often depends on the reprogramming of cell metabolism, and the metabolism reprogramming enables tumor cells to obtain the necessary nutrients from the nutrition deficient environment. Generally speaking, tumor cells metabolize glucose, fatty acids and glutamine at a much higher rate than normal cells [5]. Metabolism reprogramming is regarded as a cancer-specific characteristic, including dysregulation of glucose and glutamine metabolism, changes in lipid biosynthesis and decomposition, and so on [6,7]. In order to maintain their proliferation, tumor cells need to increase ATP production, synthesize macromolecules and reduce the generation of reducing substances or other metabolic auxiliary materials [8]. Besides, cancer cells must adapt

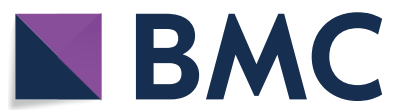

(c) The Author(s) 2021. This article is licensed under a Creative Commons Attribution 4.0 International License, which permits use, sharing, adaptation, distribution and reproduction in any medium or format, as long as you give appropriate credit to the original author(s) and the source, provide a link to the Creative Commons licence, and indicate if changes were made. The images or other third party material in this article are included in the article's Creative Commons licence, unless indicated otherwise in a credit line to the material. If material is not included in the article's Creative Commons licence and your intended use is not permitted by statutory regulation or exceeds the permitted use, you will need to obtain permission directly from the copyright holder. To view a copy of this licence, visit http://creativeco mmons.org/licenses/by/4.0/. The Creative Commons Public Domain Dedication waiver (http://creativecommons.org/publicdomain/ zero/1.0/) applies to the data made available in this article, unless otherwise stated in a credit line to the data. 
their metabolism to the dynamic changes in the process of tumor development, so as to maintain the energy level, redox status, cellular signaling and biosynthesis, thus promoting tumor growth [9]. In normal tissues, cells tend to generate energy through oxidative phosphorylation by the mitochondria, and in the absence of oxygen, glucose was catabolized to lactic acid through glycolysis. Interestingly, even in the presence of sufficient oxygen, tumor cells still use the glycolysis pathway to generate energy, in order to meet the rapid proliferation of cancer cells [10]. This process is known as the Warburg Effect. Currently, the Warburg effect has been observed in various types of tumors and has been widely accepted as a symbol of metabolic changes in cancer cells [10]. Since the discovery of the Warburg effect, the majority of researches about tumor metabolism have focused on glucose metabolism. Recently, with the deepening of the research, people are aware that other nutrients, such as amino acids, for the process of tumor cell metabolism play a key role [11].

\section{LncRNAs: a brief introduction}

Eukaryotes produce many types of RNAs, which play a crucial role in the transmission of genetic information and often exist in specific subcellular localization. The synthesis, processing and transportation of RNA are closely involved in the regulation of cell functions [12]. Less than $2 \%$ of the human genome encodes proteincoding RNA, and more than $90 \%$ of the total genome is transcribed into noncoding RNA(ncRNA) [13]. LncRNA is a kind of RNA transcript with more than 200 nucleotides, which has little protein-coding potential. It is usually transcribed by RNA polymerase II with 5 '-end cap, polyadenylation and splicing [14]. LncRNA could localize in the nucleus or cytoplasm, and the function of
lncRNA usually depends on subcellular localization [15]. Researchers have found that LncRNA was involved in multiple aspects of gene expression regulation including epigenetic, transcriptional and post-transcriptional modification. (1) LncRNA can act as an RNA decoy binding to transcription factors, thus interfering its binding to the promoter and regulating transcription [16]. (2) As a molecular sponge, adsorbing and separating miRNA from target mRNA, which affects the translational of mRNA [17]. (3) As a molecular scaffold, interacting with proteins to form the lncRNA-proteins complex, thereby regulating the protein activity or stability [18]. (4) Recruiting chromatin modifiers to reprogram chromatin [19]. (5) Binding with mRNA and affecting the translation, splicing, and stability of mRNA [20, 21] (Fig. 1). With the continuous research on lncRNA in recent years, IncRNA has been proved to be involved in tumor progression through a series of cell metabolism processes (Fig. 2), thus showing a unique advantage in tumor diagnosis, monitoring, prognosis and treatment [22]. LncRNAs can play an oncogenic or a tumor suppressive role, however, they are often dysregulated in cancers and participate in the occurrence of metabolic changes [23-25]. In this review, we mainly focus on the roles of lncRNAs in amino acid metabolism of tumor cells and discuss the pathways affected by lncRNAs in the process of cancer metabolism.

\section{Normal metabolism of amino acids}

Metabolic reprogramming enables cancer cells to adapt to increased nutrient requirements and biosynthesis, in which changes in amino acid metabolism are an important part of the metabolic reprogramming event. Amino acids, which are metabolized into proteins and converted

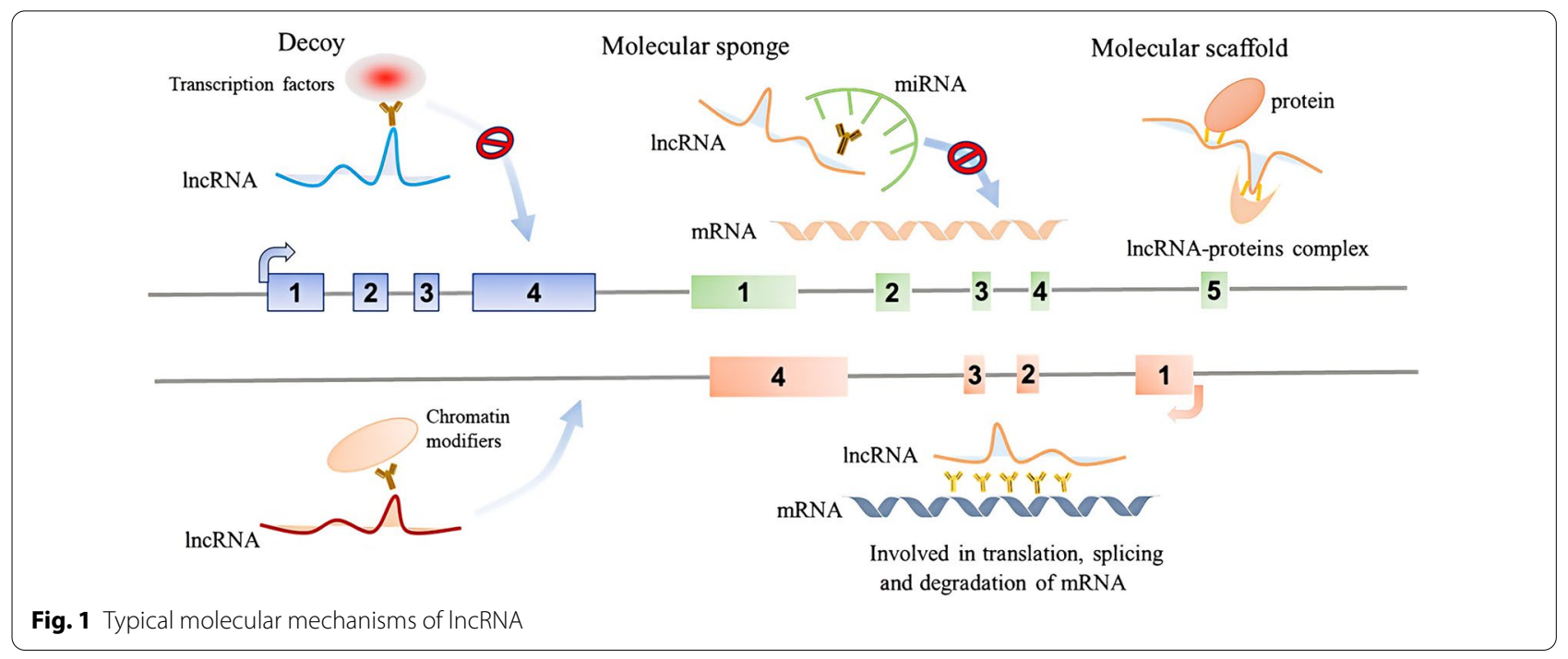




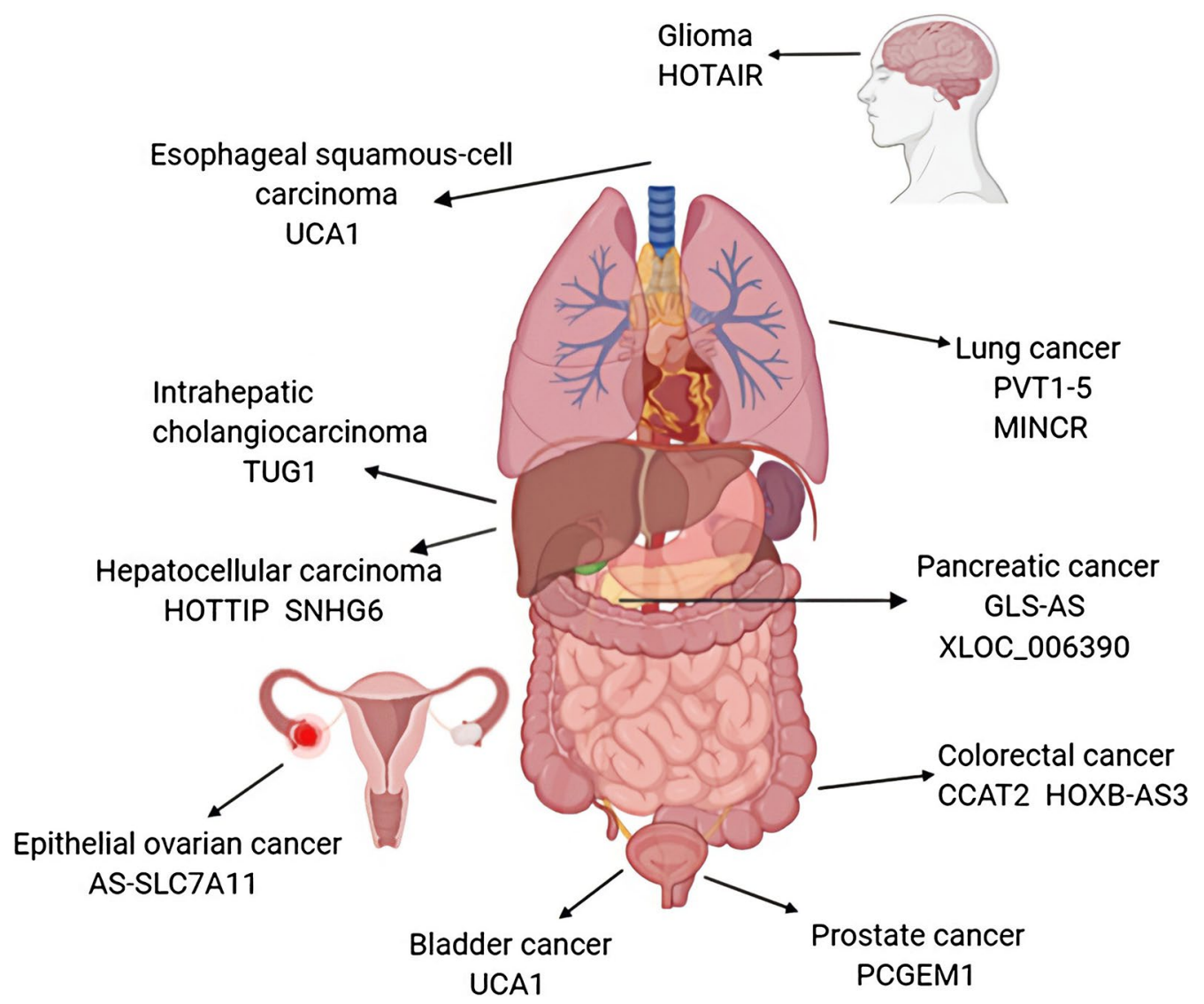

Fig. 2 Amino acid metabolism involving IncRNAs that are dysregulated in cancer

into hormones, neurotransmitters and other important nitrogen-containing active substances, are crucial for cell survival [26]. In the process of amino acid metabolism in normal cells, $1-2 \%$ of proteins are degraded every day, mostly in skeletal muscle and most of the amino acids produced by protein degradation are reused to synthesize new proteins. Intracellular protein degradation is completed by a series of enzymatic reactions [27]. Eukaryotic cells participate in the degradation of protein mainly through two pathways: (1) ATP-independent pathway in lysosomes. (2) ATP-dependent pathway in the proteasome, namely the ubiquitin-involved protein degradation process [28]. In humans, the twenty amino acids that make up proteins are usually divided into essential and non-essential amino acids. Essential amino acids are those whose carbon skeleton cannot be de novo synthesized, including isoleucine (Ile), leucine (Leu), methionine (Met), valine (Val), phenylalanine (Phe), tryptophan (Trp), histidine (His), threonine (Thr) and lysine (Lys). Semi-essential amino acids are those that can be synthesized de novo but not in sufficient quantities to maintain the normal metabolic level. Therefore, dietary supplementation is usually required. They include arginine (Arg), cysteine (Cys), glycine (Gly), glutamine (Gln), proline (Pro) and tyrosine (Tyr). Five other amino acids are considered dispensable because they are easily synthesized in vivo, including alanine (Ala), aspartic acid (Asp), asparagine (Asn), glutamate (Glu) and serine (Ser) [29]. Glutamine is the most abundant amino acid in the circulation and is second only to glucose in the metabolism of tumor cells [30]. Although glutamine is a kind of conditionally essential amino acid in vivo, it has been observed that in lots of cancers, the dependence on glutamine has unique to some cancer cells [31,32].

Amino acids are indispensable for tumor cell metabolism Mounting evidence shows that the rapid proliferation of cancer cells depends on higher demand for amino acids. A variety of amino acids play a vital function in the metabolism of tumor cells: Serine and glycine, as the basic precursors for the synthesis of proteins, nucleic acids and lipids, need more consumption to meet the rapid proliferation of tumor cells. Moreover, the biosynthesis of serine and glycine affects cellular antioxidative 
capability and also promotes tumor growth [33]; The c-MYC oncogene activates the expression of glutaminase (GLS1/GLS2) and glutamine metabolism in cancer cells, and glutamine can be converted to glutamate even in hypoxia [34]. More consumed glutamine for mitochondrial energy production is a feature of multiple cancer cell metabolism; arginine participates in the urea cycle, in which argininosuccinate synthetase (ASS) catalyzes citrulline and aspartic acid to produce argininosuccinate. Subsequently, argininosuccinate is cleaved to produce arginine. In malignant melanoma and hepatocellular carcinoma, ASS deficiency leads to a failure in arginine synthesis, and arginine deficiency interferes with the growth of cancer cells, which is used for the treatment of advanced malignant tumors [35]. Furthermore, tumor cells also preferentially uptake branched chain amino acids (BCAAs) as nutrients. The three BCAAs are valine, leucine and isoleucine. BCAA metabolism can meet some inherent requirements in the process of cancer proliferation, such as providing nitrogen for de novo nucleotide synthesis, participating in the activation of signaling pathways and influencing the expression of many crucial metabolite-derived co-factors [36]. In melanoma, for example, when leucine is deprived, hyperactivation of RAS-MEK signaling fails to inhibit mTOR, thereby triggering apoptosis of human melanoma cells [37].

\section{Glutamine metabolism: the essential part of amino acid metabolism}

Glutamine is transported into cells by solute carrier family 1 neutral amino acid transporter member 5 (SLC1A5, also known as ASCT2), and high levels of glutamine in the blood serve as a source of nitrogen and carbon for biosynthesis. Intracellular glutamine could be catalyzed by mitochondrial glutaminase to produce glutamate and ammonium ions. Glutamate is also a precursor to glutathione $(\mathrm{GSH})$, which is a major cellular antioxidant to help maintain normal immune system function [38-40]. Glutamate could be converted into $\alpha-K G$ $(\alpha$-Ketoglutarate), the intermediate product of the tricarboxylic acid (TCA) cycle, by transamination and oxidative deamination. The oxidative deamination of glutamate is catalyzed by GDH (glutamate dehydrogenase) [41]. Moreover, $\alpha-K G$ is involved in reductive carboxylation and reverse catalysis to produce citrate used for the synthesis of acetyl-CoA and lipids [42]. Oxaloacetic acid (OAA), an intermediate of the TCA cycle, is converted into aspartic acid by transamination, which is used for the synthesis of purine and pyrimidine nucleotides (Fig. 3). At the same time, glutamine can promote the synthesis of UDP-N-acetylglucosamine (UDP-GlcNAc), which plays a key role in protein folding and transport.
The lack of glutamine will lead to faulty protein folding and endoplasmic reticulum stress response [43].

\section{LncRNAs and glutaminase}

Glutamine can be catalyzed by glutaminase to produce glutamate, which is the first step of glutamine metabolism. There are two genes encoding glutaminase in mammals, namely GLS and GLS2, which show different structures and are specifically expressed in different tissues [44, 45]. Recent studies have shown that GLS is highly expressed in some malignant tumors and knockdown of GLS significantly reduces the invasion and proliferation of cancer cells, indicating the cancer-promoting role of GLS [46, 47]. GLS2 is mainly expressed in the liver and brain [48]. In some tissues, GLS2 is a target of p53 and mediates the tumor-suppressing role of p53 in cancer cells [49]. LncRNA CCAT2 (Colon Cancer Associated Transcript 2), which is located at the 8q24 amplicon of cancer risk-associated rs6983267 SNP was reported to promote the glycolysis as well as glutamine metabolism in a variety of cancers $[50,51]$. The interaction between CCAT2 and CFIm complex regulates the alternative splicing of GLS by selecting the Poly-A site of GLS pre-mRNA intron 14 and induces the production of two alternative splicing isoforms, respectively KGA(glutaminase kidney isoform) and GAC(glutaminase isoform C) [51]. Although these two isoforms have the same active site, GAC has higher catalytic activity than KGA. GAC plays a key role in mitochondrial glutamine metabolism of cancer cells, promoting cell proliferation and metastasis in vivo [52].

\section{The way IncRNAs regulate glutamine metabolism}

Previous studies have shown that miRNA can interact with specific lncRNA and degrade lncRNA at the posttranscriptional level [53]. LncRNA HOTTIP is an important oncogene of hepatocellular carcinoma (HCC). It was reported that HOTTIP was involved in GLS1-mediated glutamine metabolism in HCC, and HOTTIP overexpression could improve GLS1 expression level to enhance glutamine metabolism. HOTTIP was identified as the target of miR-192 and miR-204, which would inhibit HOTTIP expression through the Argonaute2-mediated RNA interference pathway, thereby inhibiting the proliferation of the cancer cells. GLS1, as a downstream gene of the miR-192/204-HOTTIP axis, is critical in the progression of hepatocellular carcinoma and glutamine metabolism [54].

LncRNA can interact with miRNA as an endogenous competitive RNA (ceRNA), and miRNA participates in the regulation of target gene expression by binding with the 3' UTR of target mRNA [55]. LncRNA TUG1 has been reported to promote glutamine metabolism by 


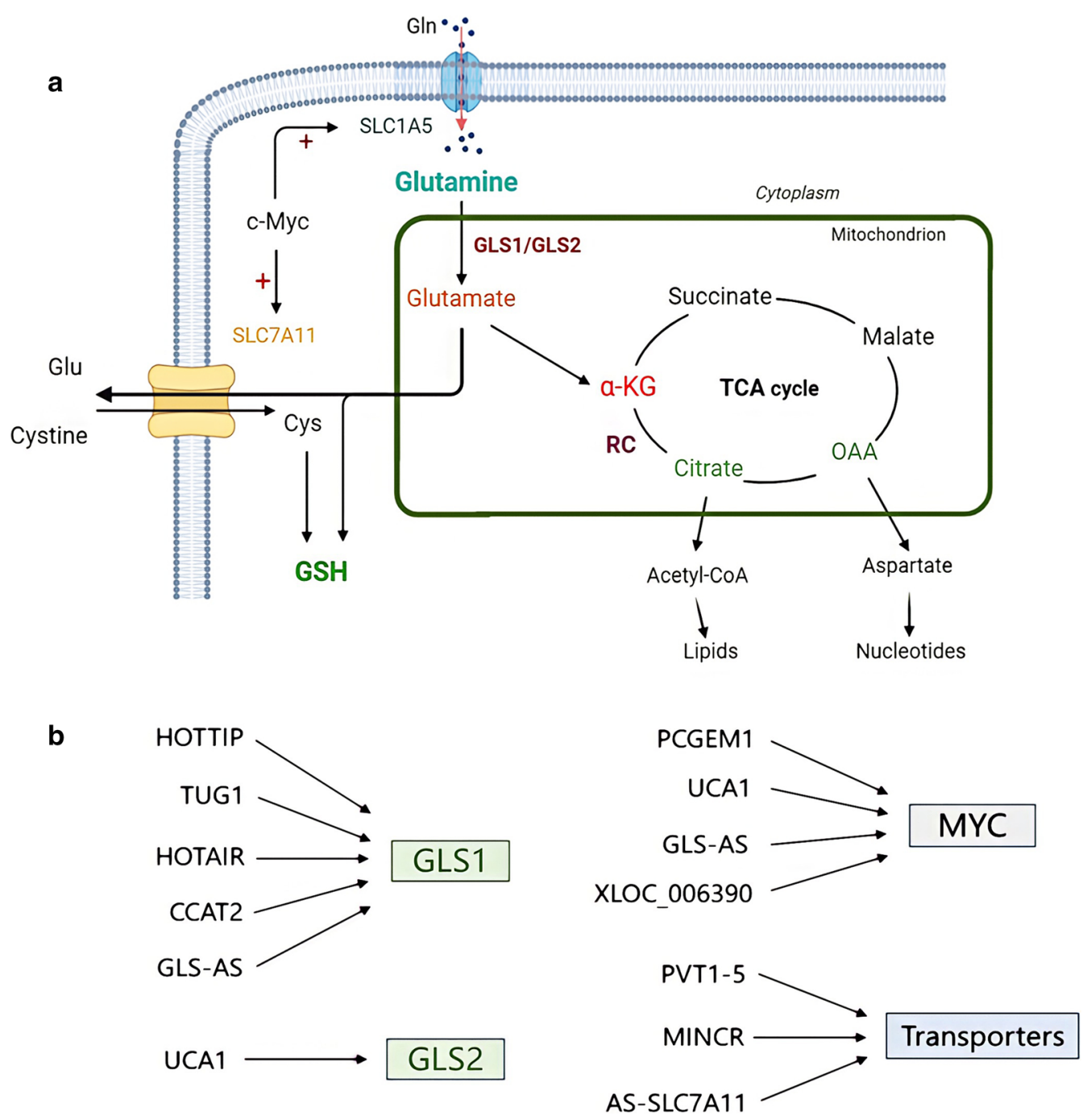

Fig. 3 a SLC1A5 transports glutamine into cells and is converted to glutamate by glutaminase, which participates in the synthesis of GSH together with intracellular Cys. SLC7A11 exchanges extracellular cystine and intracellular glutamate, and c-myc participates in the positive regulation of SLC1A5 and SLC7A11. Glutamate is converted into a-KG by L-glutamate dehydrogenase or aminotransferases, which participates in the TCA cycle. $\mathbf{a}-\mathrm{KG}$ participates in the reductive carboxylation process to produce citrate, which used for the synthesis of acetyl-CoA and lipids, aspartate produced by oxaloacetic acid transamination is a necessary substance for nucleotide synthesis. $\mathbf{b}$ LncRNAs which are involved in regulating amino acid metabolism

inhibiting miR-145 in intrahepatic cholangiocarcinoma (ICC). Sirt3, as a direct target of miR-145, has been confirmed to activate GDH in the mitochondrial matrix by deacetylation, thus positively regulating the expression of GDH [56]. LncRNA TUG1, acting as a ceRNA 'sponges' miR-145, increased the level of Sirt3 and GDH. The knockdown of TUG1 or overexpression of miR-145 resulted in a significant reduction in intracellular glutamine consumption, reduced proliferation and migration of ICC cells, and inhibited tumor development. At the same time, glutamate participates in GDH-mediated oxidative deamination which results in the reduction of $\alpha-$ KG [57]. LncRNA HOX transcript antisense intergenic RNA(HOTAIR) has a similar effect. In glioma cells, the expression of IncRNA HOTAIR is abnormally increased. Existing studies have shown that lncRNA HOTAIR as a ceRNA 'sponged' miR-126-5p and promotes glutamine metabolism in glioma. The miR-126-5p was reported to play an inhibitory role in both lung cancer and gastric cancer [58, 59]. While in glioma, GLS was confirmed to be the direct target of miR-126-5p, and miR-126-5p significantly reduced the expression of GLS in mRNA and protein levels. LncRNA HOTAIR regulated the expression of GLS through the miR-126/ 
GLS pathway, thus changing the glutamine metabolism process of glioma and promoting the development of the tumor. Glutamate was also the precursor of GSH, while miR-126-5p was negatively correlated with GSH level [60].

\section{LncRNAs mediate the antioxidant defense in cancer metabolism}

Glutamine metabolism is of great significance for maintaining the redox balance of tumor cells and the level of ROS (reactive oxygen species). GLS2 inhibits the production of ROS and mediates the antioxidant defense function of cells [61]. Glutamine can be catalyzed by GLS2 to produce glutamate, which participates in the synthesis of glutathione (GSH) in vivo. The glutathione-centered redox system is involved in the occurrence of a series of signal pathways, including the elimination of ROS, protein synthesis and cell oxidative defense functions. GSH is the most important intracellular antioxidant molecule, protecting cells from apoptosis induced by oxidative stress $[62,63]$. Glutathione mainly exists in the form of reduced glutathione (GSH) and oxidized glutathione (GSSG). Under physiological conditions, GSH is the main existing form, accounting for about $99 \%$ [64]. The ratio of GSH/GSSG reflects the redox state of cells, the lack of glutamine during cell metabolism will eliminate the effect of GLS2 on the increase of GSH levels, indicating that glutamine metabolism plays a critical role in maintaining tumor cell redox balance and ROS levels [65].

It was reported that IncRNA UCA1 participated in the malignant progression, drug resistance and metabolism reprogramming of bladder cancer [66-69]. In bladder cancer cells, UCA1 regulates the glutamine metabolism and antioxidant defense by inhibiting miR-16, which targets GLS2 for translational inhibiting and reduced GLS2 expression. This suggests a positive role of UCA1 in reducing $\mathrm{ROS}$ and sustaining the redox balance of cancer cells. Notably, the mRNA level of UCA1 and GLS2 are positively correlated, and the expression of GLS2 is negatively correlated to the miR-16 in bladder cancer.

\section{The interplay between IncRNAs and MYC}

The MYC proto-oncogene family includes c-Myc, $\mathrm{N}-\mathrm{Myc}, \mathrm{L}-\mathrm{Myc}$, which are involved in the occurrence of various human tumors [70]. Cancer cells maintain their rapid proliferation and metastasis through metabolic reprogramming. Studies on PI3K/AKT/mTOR signaling pathway showed that cancer cells were strongly addicted to glucose and other nutrients such as amino acids. Glutamine, as one of the major energy substrate of cancer cells, could also easily lead to addiction [71]. Metabolic reprogramming induced by MYC leads to glutamine addiction, its high expression can induce the glutamine transporter, glutaminase and lactate dehydrogenase A (LDH-A) expression. Myc is a major regulator of glutamine metabolism [72]. Cancer cells accelerate mitochondrial glutaminolysis by Myc, which provides cells with fast-generating NADPH [73]. NADPH participates in many metabolic reactions, such as the synthesis of fatty acids, cholesterol and non-essential amino acids, and plays a key role in maintaining the reduction state of GSH [74].

As we all know, the androgen receptor (AR) signaling plays an important role in the progression of prostate cancer [75]. LncRNA PCGEM1 (prostate cancer gene expression marker 1) is an androgen-induced prostatespecific IncRNA [76], which has been confirmed to regulate the metabolism including the tricarboxylic acid cycle, glutamine metabolism and pentose phosphate pathway of prostate cancer by activating c-Myc. C-Myc recruits PCGEM1 to the promoter of its target genes, which promotes chromatin recruitment and enhances its transactivation activity. When endogenous PCGEM1 is knocked down, the activity of c-Myc will decrease, indicating that PCGEM1 plays a metabolic regulation role as a coactivator of c-Myc in prostate cancer cells. This is also the first report that lncRNA binds with c-Myc and acts as a co-activator to regulate metabolic reprogramming [77]. In esophageal squamous cell carcinoma (ESCC), the Wnt/ $\beta$-catenin signaling pathway is highly correlated with the progression of ESCC, and its abnormal activation can lead to the occurrence of a variety of cancers [78]. While c-Myc, as a target gene of the Wnt/ $\beta$-catenin signaling pathway, is involved in regulating ESCC cell cycle distribution and promoting tumor progression. The overexpression of UCA1 reduced the level of c-Myc and the $\beta$-catenin protein in the nucleus, and the proliferation and invasion of ESCC cells were obviously inhibited [79]. On the other hand, pancreatic cancer (PC) is a group of malignant tumors that mainly originated from the pancreatic ductal epithelium and acinar cells, which is extremely malignant and progresses rapidly [80]. Recent studies have found that antisense lncRNA of glutaminase (GLS-AS) is involved in the pathogenesis of pancreatic cancer by mediating the mutual feedback of Myc and GLS in the tumor nutrients stress microenvironment. Tumor nutrients stress microenvironment is a critical factor for the downregulation of GLS-AS in pancreatic cancer, and GLS is the key target of GLS-AS. GLS-AS forms double-stranded RNA with GLS pre-mRNA and inhibits GLS expression at the post-transcriptional level. The promoter region of GLS-AS contains the binding site of Myc. As a multifunctional transcription factor, Myc down-regulates the expression of GLS-AS by inhibiting its transcriptional activity [81]. C-Myc is involved in regulating a variety of signaling pathways in cancer cells, 
and recent studies have shown that c-Myc participates in the regulation of glutamine metabolism by mediating the transcription of GDH. LncRNA XLOC_006390 was also found to promote pancreatic cancer through regulating amino acid metabolism. XLOC_006390 promotes the stability of c-Myc by preventing its ubiquitination and subsequently upregulates GDH. GDH was confirmed to be generally up-regulated in cancer, and knockdown of GDH in cancer cells can significantly attenuate the glutaminolysis rate [82]. As GDH is closely related to pancreatic cancer progression, reduce the expression of GDH via the XLOC_006390/c-Myc/GDH signal axis, leading to the lower glutamine metabolism level, thus may providing a new vision for pancreatic cancer therapy [83].

\section{LncRNAs mediate amino acid transporters' function in cancer}

The proliferation of tumor cells is characterized by uncontrolled and rapid division. Amino acids, as a class of major nutrients, are very important for the growth of tumor cells [84]. Due to excessive nutrient requirements, some amino acid transporters are up-regulated in the development of cancer. Four amino acid transporters have been found to be highly expressed in cancer, namely SLC7A5, SLC7A11, SLC1A5 and SLC6A14. SLC7A5 is also referred to as the LAT1 (L-Amino acid transporter 1), with a high affinity to the branched-chain and neutral amino acid. It mediates a sodium-independent mandatory exchange and this exchange mechanism allows a large number of neutral amino acids to be balanced on the membrane [85]. The promoter of SLC7A5 has a typical binding site with $\mathrm{C}-\mathrm{Myc}$, and the high expression of C-Myc often leads to an increase of SLC7A5 expression level in cancer cells [86]. It was reported that SLC7A5 was closely related to cell proliferation in lung cancer. SLC7A5 has been proved to be the direct target of miR-126 [87], which significantly inhibits the transport of other amino acids such as leucine, thus affecting the amino acid metabolism and the activation of the mTOR signal $[88,89]$. The involvement of leucine in mTOR signal activation has been widely recognized [90]. Several lncRNAs were reported to regulate the amino acids metabolism through miR-126. For example, lncRNA PVT1-5 (Plasmacytoma variant translocation 1-5) was reported to be up-regulated and promoted the progression of lung cancer through sponging miR-126 [91]. Moreover, in non-small cell lung cancer (NSCLC), MYC induced long noncoding RNA (MINCR) is significantly up-regulated and promoted the proliferation and migration of NSCLC cells through targeting miR-126 [92].

Cysteine, as a rate-limiting amino acid for GSH synthesis, whose level affects GSH balance in cells, is considerable for the sustaining of redox balance of cells. SLC7A11, the major transporter for the exchange of extracellular cysteine and intracellular glutamate, protects cancer cells from apoptosis and promoting tumor development by improving the synthesis of GSH [93]. Antisense lncRNA AS-SLC7A11 is significantly reduced in epithelial ovarian cancer (EOC) and has been proved to inhibit SLC7A11 expression in ovarian cancer. The knockdown of ASSLC7A11 increases the expression of SLC7A11 and improves the proliferation and viability of ovarian cancer cells [94].

\section{Other mechanisms of IncRNAs in amino acid metabolism}

The role of lncRNAs in cancer is diverse, recent studies have shown that polypeptides encoded by lncRNA can also suppress cancer in amino acid metabolism [95]. LncRNA HOXB-AS3 can encode a conserved peptide containing 53 amino acids. This peptide inhibits amino acid metabolism and glycolysis to slow the progression of colon cancer [96]. As we know, the methionine cycle elucidates the metabolism of sulfur-containing amino acids in vivo. Methionine adenosyltransferases (MAT) catalyze the methionine cycle to produce S-adenosylmethionine (SAMe). MAT has two coding genes in vivo, respectively MAT1A and MAT2A, and in the liver, their regulation modes for the SAMe are completely opposite: MAT1A upregulates the concentration of the SAMe, while MAT2A downregulates the SAMe [97, 98]. The methyl in SAMe is called active methyl, which makes SAMe to be the most important direct donor of methyl in vivo [99]. In hepatocellular carcinoma (HCC), lncRNA SNHG6, as a molecular sponge of miR-1297, is involved in regulating genome-wide methylation levels. MiR-1297 directly binds to the 3 'UTR of the MAT2A mRNA, leading to its translational inhibition. SNHG6 upregulates the expression of MAT2A, thereby negatively regulating the concentration of SAMe in cells, leading to significant genome-wide hypomethylation of hepatocellular carcinoma, which is a major feature of tumor genesis [100].

\section{Conclusions}

LncRNA in malignant tumors is widely involved in the process of metabolism [101]. Besides involved in the regulation of glycolysis and lipid metabolism [102], lncRNA was also found to be involved in the process of amino acid metabolism: regulate the action mode of amino acid transporters, leading to the lack of several amino acid types; as a ceRNA to interact with miRNA involved in regulating glutamine metabolism; control alternative splicing of glutaminase to regulate metabolic processes in vivo; reduce intracellular ROS level so as to protect the function of the mitochondrion and regulate antioxidant defense in cells; encode some peptides to play a role in the anticancer properties, which 
can enlighten some potential ideas for treatment; play a unique role in the regulation of signaling pathways in Myc-driven cancers. In this review, we highlighted the characteristics of lncRNAs in regulating cancer amino acid metabolism, and the change of glutamine metabolism played a vital role in the process of oncogenesis [103]. Tumor cells seem to be very dependent on glutamine so that we can reasonably infer that drugs act on glutamine metabolism can play an unexpected role in the process of cancer development. The rediscovery on the effect of glutamine in tumor cells may provide us with promising clinical treatment. Besides, the metabolic mechanism of lncRNA in cancer also needs to further elucidate, which will be a great help to find new biomarkers and therapeutic targets in cancer treatment. There are many other lncRNAs related to cancer cell metabolism, whose structures and functions are not clear to us. Given that the research on lncRNAs is still at the preliminary stage, it seems promising to discover novel lncRNAs and develop lncRNA-based targeted therapeutic strategies. The study afterward will be full of challenges and opportunities.

\begin{abstract}
Abbreviations
IncRNAs : Long non-coding RNAs; ncRNA: Noncoding RNA; ceRNA: Endogenous competitive RNA; GLS: Glutaminase; KGA: Glutaminase kidney isoform; GAC: Glutaminase isoform C; GSH: Glutathione or reduced glutathione; GSSG: Oxidized glutathione; a-KG: a-Ketoglutarate; ASS: Argininosuccinate synthetase; GDH: Glutamate dehydrogenase; ROS: Reactive oxygen species; LDH-A: Lactate dehydrogenase A; TCA cycle: Tricarboxylic acid cycle; MAT: Methionine adenosyltransferases; Ile: Isoleucine; Leu: Leucine; Met: Methionine; Val: Valine; Phe: Phenylalanine; Trp: Tryptophan; His: Histidine; Thr: Threonine; Lys: Lysine; Arg: Arginine; Cys: Cysteine; Gly: Glycine; Gln: Glutamine; Pro: Proline; Tyr: Tyrosine; Ala: Alanine; Asp: Aspartic acid; Asn: Asparagine; Glu: Glutamate; Ser: Serine; OAA: Oxaloacetic acid; BCAAs: Branched chain amino acids; SLC1A5: Solute carrier family 1 neutral amino acid transporter member 5; LAT1: L-Amino acid transporter 1; UDP-GIcNAc: UDP-N-acetylglucosamine; AR: Androgen receptor; SAMe: S-adenosylmethionine; CCAT2: Colon Cancer Associated Transcript 2; HOTAIR: HOX transcript antisense intergenic RNA; PVT1-5: Plasmacytoma variant translocation 1-5; MINCR: MYC induced long noncoding RNA; PCGEM1: Prostate cancer gene expression marker 1; GLS-AS: Antisense IncRNA of glutaminase; HCC: Hepatocellular carcinoma; ICC: Intrahepatic cholangiocarcinoma; PC: Pancreatic cancer; NSCLC: Non-small cell lung cancer; ESCC: Esophageal squamous cell carcinoma; EOC: Epithelial ovarian cancer.
\end{abstract}

\section{Acknowledgements}

Not applicable.

\section{Authors' contributions}

YHG performed the study and drafted the manuscript together with BL. RFL, ZZD and FFZ performed the literature search and screen. YPL, BY and DZ conceived and designed the study. XBL and ZPZ supervised the study and revised the manuscript. All authors read and approved the final manuscript.

\section{Funding}

This study was supported by the National Natural Science Foundation of China (No. 81672866, 81960501 and 81560452 to XBL); the Natural Science Foundation of Jiangxi Province (No. 20161BAB205192 and 20171ACB21073 to $X B L$ ); the Natural Science Foundation of Jiangxi Province the Youth Science Foundation of Jiangxi Province (No.20122BAB215028 to ZPZ).

\section{Availability of data and materials}

The material supporting the conclusion of this review has been included within the article.

\section{Declarations}

Ethics approval and consent to participate

Not applicable.

\section{Consent for publication}

Not applicable.

\section{Competing interests}

The authors declare that they have no competing interests.

\section{Author details}

1 Jiangxi Key Laboratory of Cancer Metastasis and Precision Treatment, The Third Affiliated Hospital of Nanchang University, Northern 128 Xiangshan Road, Nanchang 330008, Jiangxi, People's Republic of China. ${ }^{2}$ Department of Orthopedics, The Third Affiliated Hospital of Nanchang University, Northern 128 Xiangshan Road, Nanchang 330008, Jiangxi, People's Republic of China. ${ }^{3}$ Nanchang Key Laboratory of Orthopaedics, The Third Affiliated Hospital of Nanchang University, Nanchang, China. ${ }^{4}$ Medical Department of Graduate School, Nanchang University, Nanchang 330006, Jiangxi, China.

Received: 29 January 2021 Accepted: 7 April 2021

Published online: 13 April 2021

\section{References}

1. Zhu J, Thompson CB. Metabolic regulation of cell growth and proliferation. Nat Rev Mol Cell Biol. 2019;20(7):436-50.

2. Janku F, Yap TA, Meric-Bernstam F. Targeting the PI3K pathway in cancer: are we making headway? Nat Rev Clin Oncol. 2018;15(5):273-91.

3. Carling D. AMPK signalling in health and disease. Curr Opin Cell Biol. 2017:45:31-7.

4. Cairns RA, Harris IS, Mak TW. Regulation of cancer cell metabolism. Nat Rev Cancer. 2011;11(2):85-95.

5. Pavlova NN, Thompson CB. The emerging hallmarks of cancer metabolism. Cell Metab. 2016;23(1):27-47.

6. Daye D, Wellen KE. Metabolic reprogramming in cancer: unraveling the role of glutamine in tumorigenesis. Semin Cell Dev Biol. 2012;23(4):362-9.

7. Li Z, Zhang H. Reprogramming of glucose, fatty acid and amino acid metabolism for cancer progression. Cell Mol Life Sci. 2016;73(2):377-92.

8. Lunt SY, Vander MG, Heiden. Aerobic glycolysis: meeting the metabolic requirements of cell proliferation. Annu Rev Cell Dev Biol. 2011;27:441-64.

9. Lehuede $C$, et al. Metabolic plasticity as a determinant of tumor growth and metastasis. Cancer Res. 2016;76(18):5201-8.

10. Potter M, Newport E, Morten KJ. The Warburg effect: 80 years on. Biochem Soc Trans. 2016;44(5):1499-505.

11. DeBerardinis RJ, Cheng T. Q's next: the diverse functions of glutamine in metabolism, cell biology and cancer. Oncogene. 2010;29(3):313-24.

12. Djebali $\mathrm{S}$, et al. Landscape of transcription in human cells. Nature. 2012:489(7414):101-8.

13. Consortium EP. An integrated encyclopedia of DNA elements in the human genome. Nature. 2012;489(7414):57-74.

14. Quinn JJ, Chang HY. Unique features of long non-coding RNA biogenesis and function. Nat Rev Genet. 2016;17(1):47-62.

15. Chen LL. Linking long noncoding RNA localization and function. Trends Biochem Sci. 2016;41(9):761-72.

16. Yang $L$, et al. IncRNA-dependent mechanisms of androgen-receptorregulated gene activation programs. Nature. 2013;500(7464):598-602.

17. Thomson DW, Dinger ME. Endogenous microRNA sponges: evidence and controversy. Nat Rev Genet. 2016;17(5):272-83.

18. Munschauer $M$, et al. The NORAD IncRNA assembles a topoisomerase complex critical for genome stability. Nature. 2018;561(7721):132-6. 
19. Wang KC, Chang HY. Molecular mechanisms of long noncoding RNAs. Mol Cell. 2011;43(6):904-14.

20. Schmitt AM, Chang HY. Long noncoding RNAs in cancer pathways. Cancer Cell. 2016;29(4):452-63.

21. Guil S, Esteller M. RNA-RNA interactions in gene regulation: the coding and noncoding players. Trends Biochem Sci. 2015;40(5):248-56.

22. Liu H, et al. Long non-coding RNAs involved in cancer metabolic reprogramming. Cell Mol Life Sci. 2019;76(3):495-504.

23. Li M, et al. InCRNA-CYTOR works as an oncogene through the CYTOR/miR-3679-5p/MACC1 Axis in colorectal cancer. DNA Cell Biol. 2019;38(6):572-82.

24. Cho SW, et al. Promoter of IncRNA gene PVT1 Is a tumor-suppressor DNA boundary element. Cell. 2018;173(6):1398-412 e22.

25. Chi Y, et al. Long non-coding RNA in the pathogenesis of cancers. Cells. 2019;8(9):1015.

26. Jones $\mathrm{CL}$, et al. Inhibition of amino acid metabolism selectively targets human leukemia stem cells. Cancer Cell. 2018;34(5):724-40 e4.

27. Saftig P, Klumperman J. Lysosome biogenesis and lysosomal membrane proteins: trafficking meets function. Nat Rev Mol Cell Biol. 2009;10(9):623-35.

28. Hochstrasser M. Ubiquitin, proteasomes, and the regulation of intracellular protein degradation. Curr Opin Cell Biol. 1995;7(2):215-23.

29. Fernandes HS, et al. Amino acid deprivation using enzymes as a targeted therapy for cancer and viral infections. Expert Opin Ther Pat. 2017;27(3):283-97.

30. Cluntun AA, et al. Glutamine metabolism in cancer: understanding the heterogeneity. Trends Cancer. 2017;3(3):169-80.

31. Vander Heiden MG. Targeting cancer metabolism: a therapeutic window opens. Nat Rev Drug Discov. 2011;10(9):671-84.

32. Tennant DA, Duran RV, Gottlieb E. Targeting metabolic transformation for cancer therapy. Nat Rev Cancer. 2010;10(4):267-77.

33. Amelio I, et al. Serine and glycine metabolism in cancer. Trends Biochem Sci. 2014;39(4):191-8.

34. Le A, et al. Glucose-independent glutamine metabolism via TCA cycling for proliferation and survival in B cells. Cell Metab. 2012;15(1):110-21.

35. Feun LG, Kuo MT, Savaraj N. Arginine deprivation in cancer therapy. Curr Opin Clin Nutr Metab Care. 2015;18(1):78-82.

36. Sivanand S, Vander Heiden MG. Emerging roles for branched-chain amino acid metabolism in cancer. Cancer Cell. 2020;37(2):147-56.

37. Sheen $\mathrm{JH}$, et al. Defective regulation of autophagy upon leucine deprivation reveals a targetable liability of human melanoma cells in vitro and in vivo. Cancer Cell. 2011;19(5):613-28.

38. DeBerardinis RJ, Chandel NS. Fundamentals of cancer metabolism. Sci Adv. 2016;2(5):e1600200.

39. Altman BJ, Stine ZE, Dang CV. From Krebs to clinic: glutamine metabolism to cancer therapy. Nat Rev Cancer. 2016;16(10):619-34.

40. Forman $\mathrm{HJ}$, Zhang $\mathrm{H}$, Rinna A. Glutathione: overview of its protective roles, measurement, and biosynthesis. Mol Aspects Med. 2009;30(1-2):1-12.

41. Smith $\mathrm{HQ}$, et al. Glutamate dehydrogenase, a complex enzyme at a crucial metabolic branch point. Neurochem Res. 2019;44(1):117-32.

42. Ward PS, et al. The common feature of leukemia-associated IDH1 and $\mathrm{IDH} 2$ mutations is a neomorphic enzyme activity converting alphaketoglutarate to 2-hydroxyglutarate. Cancer Cell. 2010;17(3):225-34.

43. Wellen $\mathrm{KE}$, et al. The hexosamine biosynthetic pathway couples growth factor-induced glutamine uptake to glucose metabolism. Genes Dev. 2010;24(24):2784-99.

44. Mates JM, et al. Glutaminase isoenzymes as key regulators in metabolic and oxidative stress against cancer. Curr Mol Med. 2013;13(4):514-34.

45. Mates JM, Campos-Sandoval JA, Marquez J. Glutaminase isoenzymes in the metabolic therapy of cancer. Biochim Biophys Acta Rev Cancer. 2018;1870(2):158-64.

46. Pan T, et al. Elevated expression of glutaminase confers glucose utilization via glutaminolysis in prostate cancer. Biochem Biophys Res Commun. 2015;456(1):452-8.

47. Consortium GT. Human genomics. The genotype-tissue expression (GTEx) pilot analysis: multitissue gene regulation in humans. Science. 2015;348(6235):648-60.

48. Campos-Sandoval JA, et al. Expression of functional human glutaminase in baculovirus system: affinity purification, kinetic and molecular characterization. Int J Biochem Cell Biol. 2007;39(4):765-73.
49. Suzuki S, et al. Phosphate-activated glutaminase (GLS2), a p53-inducible regulator of glutamine metabolism and reactive oxygen species. Proc Natl Acad Sci U S A. 2010;107(16):7461-6.

50. Tomlinson I, et al. A genome-wide association scan of tag SNPS identifies a susceptibility variant for colorectal cancer at 8q24.21. Nat Genet. 2007; 39(8): 984-8.

51. Redis RS, et al. Allele-specific reprogramming of cancer metabolism by the long non-coding RNA CCAT2. Mol Cell. 2016;61(4):520-34.

52. Cassago A, et al. Mitochondrial localization and structure-based phosphate activation mechanism of Glutaminase $C$ with implications for cancer metabolism. Proc Natl Acad Sci U S A. 2012;109(4):1092-7.

53. Liu Q, et al. LncRNA loc285194 is a p53-regulated tumor suppressor. Nucleic Acids Res. 2013;41(9):4976-87.

54. Ge Y, et al. MiRNA-192 [corrected] and miRNA-204 directly suppress IncRNA HOTTIP and interrupt GLS1-mediated glutaminolysis in hepatocellular carcinoma. PLoS Genet. 2015;11(12):e1005726.

55. Tay Y, Rinn J, Pandolfi PP. The multilayered complexity of ceRNA crosstalk and competition. Nature. 2014;505(7483):344-52.

56. Schlicker $C$, et al. Substrates and regulation mechanisms for the human mitochondrial sirtuins Sirt3 and Sirt5. J Mol Biol. 2008;382(3):790-801.

57. Zeng $B$, et al. LncRNA TUG1 sponges miR-145 to promote cancer progression and regulate glutamine metabolism via Sirt3/GDH axis. Oncotarget. 2017;8(69):113650-61.

58. An Q, et al. Matrine induces cell cycle arrest and apoptosis with recovery of the expression of miR-126 in the A549 non-small cell lung cancer cell line. Mol Med Rep. 2016;14(5):4042-8.

59. Wang J, et al. Regulator of G-protein signaling 3 targeted by miR-126 correlates with poor prognosis in gastric cancer patients. Anticancer Drugs. 2017;28(2):161-9.

60. Liu L, et al. Long non-coding RNA HOTAIR acts as a competing endogenous RNA to promote glioma progression by sponging miR-126-5p. J Cell Physiol. 2018; 233(9): 6822-6831.

61. Zhang J, et al. Epigenetic silencing of glutaminase 2 in human liver and colon cancers. BMC Cancer. 2013;13:601.

62. $\mathrm{Lv} \mathrm{H}$, et al. Unraveling the potential role of glutathione in multiple forms of cell death in cancer therapy. Oxid Med Cell Longev. 2019;2019:3150145.

63. Sies H. Glutathione and its role in cellular functions. Free Radic Biol Med. 1999;27(9-10):916-21.

64. Lu SC. Glutathione synthesis. Biochim Biophys Acta. 2013;1830(5):3143-53.

65. D'Autreaux B, Toledano MB. ROS as signalling molecules: mechanisms that generate specificity in ROS homeostasis. Nat Rev Mol Cell Biol. 2007:8(10):813-24.

66. Yang $\mathrm{C}$, et al. Long non-coding RNA UCA1 regulated cell cycle distribution via CREB through PI3-K dependent pathway in bladder carcinoma cells. Gene. 2012;496(1):8-16.

67. Wang F, et al. UCA1, a non-protein-coding RNA up-regulated in bladder carcinoma and embryo, influencing cell growth and promoting invasion. FEBS Lett. 2008;582(13):1919-27.

68. Jiang $\mathrm{M}$, et al. Corrigendum to "A novel long non-coding RNA-ARA: Adriamycin resistance-associated" [Biochem. Pharmacol. 87 (2014) 254-283]. Biochem Pharmacol, 2020. 172: 113732.

69. Han Y, et al. UCA1, a long non-coding RNA up-regulated in colorectal cancer influences cell proliferation, apoptosis and cell cycle distribution. Pathology. 2014;46(5):396-401.

70. Carroll PA, et al. Deregulated Myc requires MondoA/MIx for metabolic reprogramming and tumorigenesis. Cancer Cell. 2015;27(2):271-85.

71. Bhutia YD, et al. Amino Acid transporters in cancer and their relevance to "glutamine addiction": novel targets for the design of a new class of anticancer drugs. Cancer Res. 2015;75(9):1782-8.

72. Dang CV. Rethinking the Warburg effect with Myc micromanaging glutamine metabolism. Cancer Res. 2010;70(3):859-62.

73. Wise $D R$, et al. Myc regulates a transcriptional program that stimulates mitochondrial glutaminolysis and leads to glutamine addiction. Proc Natl Acad Sci U S A. 2008;105(48):18782-7.

74. Stanton RC. Glucose-6-phosphate dehydrogenase, NADPH, and cell survival. IUBMB Life. 2012;64(5):362-9.

75. Dai C, Heemers H, Sharifi N. Androgen signaling in prostate cancer. Cold Spring Harb Perspect Med. 2017;7(9):a030452. 
76. Srikantan V, et al. PCGEM1, a prostate-specific gene, is overexpressed in prostate cancer. Proc Natl Acad Sci U S A. 2000;97(22):12216-21.

77. Hung $\mathrm{CL}$, et al. A long noncoding RNA connects C-Myc to tumor metabolism. Proc Natl Acad Sci U S A. 2014;111(52):18697-702.

78. Wang W, Xue L, Wang P. Prognostic value of beta-catenin, c-myc, and cyclin D1 expressions in patients with esophageal squamous cell carcinoma. Med Oncol. 2011:28(1):163-9.

79. Wang $X$, et al. IncRNA UCA1 inhibits esophageal squamous-cell carcinoma growth by regulating the Wnt signaling pathway. J Toxicol Environ Health A. 2016;79(9-10):407-18.

80. Zhu H, et al. Pancreatic cancer: challenges and opportunities. BMC Med. 2018;16(1):214.

81. Li B, Simon MC. Molecular pathways: targeting MYC-induced metabolic reprogramming and oncogenic stress in cancer. Clin Cancer Res. 2013;19(21):5835-41.

82. Jin L, et al. Glutamate dehydrogenase 1 signals through antioxidant glutathione peroxidase 1 to regulate redox homeostasis and tumor growth. Cancer Cell. 2015;27(2):257-70.

83. He J, et al. LnCRNA XLOC_006390 promotes pancreatic carcinogenesis and glutamate metabolism by stabilizing c-Myc. Cancer Lett. 2020;469:419-28.

84. Fung MKL, Chan GC. Drug-induced amino acid deprivation as strategy for cancer therapy. J Hematol Oncol. 2017;10(1):144.

85. Fotiadis D, Kanai Y, Palacin M. The SLC3 and SLC7 families of amino acid transporters. Mol Aspects Med. 2013;34(2-3):139-58.

86. Gao P, et al. c-Myc suppression of miR-23a/b enhances mitochondrial glutaminase expression and glutamine metabolism. Nature. 2009;458(7239):762-5.

87. Miko E, et al. miR-126 inhibits proliferation of small cell lung cancer cells by targeting SLC7A5. FEBS Lett. 2011;585(8):1191-6.

88. Nicklin $\mathrm{P}$, et al. Bidirectional transport of amino acids regulates mTOR and autophagy. Cell. 2009;136(3):521-34.

89. Jewell $\mathrm{J}$, et al. Metabolism. Differential regulation of mTORC1 by leucine and glutamine. Science. 2015;347(6218):194-8.

90. Son SM, et al. Leucine signals to mTORC1 via Its metabolite acetylcoenzyme A. Cell Metab. 2019;29(1):192-201 e7.

91. Li H, et al. Long non-coding RNA PVT1-5 promotes cell proliferation by regulating miR-126/SLC7A5 axis in lung cancer. Biochem Biophys Res Commun. 2018;495(3):2350-5.
92. Wang J, et al. Up-regulation of long noncoding RNA MINCR promotes non-small cell of lung cancer growth by negatively regulating miR-126/ SLC7A5 axis. Biochem Biophys Res Commun. 2019;508(3):780-4.

93. Lewerenz J, et al. The cystine/glutamate antiporter system $\mathrm{x}(\mathrm{c})(-)$ in health and disease: from molecular mechanisms to novel therapeutic opportunities. Antioxid Redox Signal. 2013;18(5):522-55.

94. Yuan J, Liu Z, Song R. Antisense IncRNA As-SLC7A11 suppresses epithelial ovarian cancer progression mainly by targeting SLC7A11. Pharmazie. 2017;72(7):402-7.

95. Storz G, Wolf Yl, Ramamurthi KS. Small proteins can no longer be ignored. Annu Rev Biochem. 2014;83:753-77.

96. Huang JZ, et al. A peptide encoded by a putative IncRNA HOXB-AS3 suppresses colon cancer growth. Mol Cell. 2017;68(1):171-84 e6.

97. Avila MA, et al. Reduced mRNA abundance of the main enzymes involved in methionine metabolism in human liver cirrhosis and hepatocellular carcinoma. J Hepatol. 2000;33(6):907-14.

98. Martinez-Chantar ML, et al. Methionine adenosyltransferase II beta subunit gene expression provides a proliferative advantage in human hepatoma. Gastroenterology. 2003;124(4):940-8.

99. Lu SC. S-Adenosylmethionine. Int J Biochem Cell Biol. 2000;32(4):391-5.

100. Guo T, et al. SNHG6 acts as a genome-wide hypomethylation trigger via coupling of miR-1297-mediated S-adenosylmethionine-dependent positive feedback loops. Cancer Res. 2018;78(14):3849-64.

101. Huarte M. The emerging role of IncRNAs in cancer. Nat Med. 2015:21(11):1253-61.

102. Chen F, et al. Extracellular vesicle-packaged HIF-1alpha-stabilizing IncRNA from tumour-associated macrophages regulates aerobic glycolysis of breast cancer cells. Nat Cell Biol. 2019;21(4):498-510.

103. Wang Y, et al. Coordinative metabolism of glutamine carbon and nitrogen in proliferating cancer cells under hypoxia. Nat Commun. 2019;10(1):201.

\section{Publisher's note}

Springer Nature remains neutral with regard to jurisdictional claims in published maps and institutional affiliations.

Ready to submit your research? Choose BMC and benefit from:

- fast, convenient online submission

- thorough peer review by experienced researchers in your field

- rapid publication on acceptance

- support for research data, including large and complex data types

- gold Open Access which fosters wider collaboration and increased citations

- maximum visibility for your research: over $100 \mathrm{M}$ website views per year

At $\mathrm{BMC}$, research is always in progress.

Learn more biomedcentral.com/submissions 